\title{
COVID-19 Data Visualization through Automatic Phase Detection
}

\author{
PRADIPTA BISWAS, KAMALPREET SINGH SALUJA, SOMNATH ARJUN, LRD MURTHY, \\ GOWDHAM PRABHAKAR, VINAY KRISHNA SHARMA, and JEEVITHA SHREE DV, \\ Centre for Product Design and Manufacturing, Indian Institute of Science, Bangalore, India
}

\begin{abstract}
This article presents a new interactive visualization system to represent and compare rate of spread of COVID-19 pandemic across different countries over time. We surveyed existing visualization techniques used in various websites and media outlets and introduced use of a knee detection algorithm that divides the exponential spread in multiple linear components. A set of use cases demonstrates the utility of the system in comparing the spread across different countries for both analysis and prediction.
\end{abstract}

CCS Concepts: • Human-centered computing $\rightarrow$ Visualization techniques; User interface design;

Additional Key Words and Phrases: Information visualization, data science, COVID-19, knee detection

ACM Reference format:

Pradipta Biswas, KamalPreet Singh Saluja, Somnath Arjun, LRD Murthy, Gowdham Prabhakar, Vinay Krishna Sharma, and Jeevitha Shree DV. 2020. COVID-19 Data Visualization through Automatic Phase Detection. Digit. Gov.: Res. Pract. 1, 4, Article 25 (July 2020), 8 pages.

https://doi.org/10.1145/3411756

\section{INTRODUCTION}

Data visualization or information visualization always played a key role in scientific analysis. Starting from John Snow's analysis of cholera epidemic in 1854 to now, a good visualization can represent inherent trends in data that may not otherwise be visible from raw numbers. The recent COVID-19 pandemic poses new challenges to data scientists, too, for its vast and rapid spread and significant economic impact. This article investigates existing visualization techniques used to represent ongoing spread of the pandemic and then proposes a new web-based interactive visualization tool (http://cambum.net/Covid19/index.html). In particular, we have developed and deployed a web-based interactive software that indicates spread of COVID-19 pandemic by automatically dividing the duration of spread based on rate of increase of cases. It uses a knee detection algorithm to progressively divide the duration. The shape of the graphs (such as linear, parabolic, or exponential) can be compared at different stages and countries with respect to the average number of new cases and deaths. Instead of dividing the

Authors' addresses: P. Biswas, K. S. Saluja, S. Arjun, LRD Murthy, G. Prabhakar, V. K. Sharma, and J. Shree DV, Centre for Product Design and Manufacturing (CPDM), Indian Institute of Science, Bengaluru, India; emails: \{pradipta, kamalpreets, somnatharjun, lrdmurthy, gowdhamp, vinaysharma, jeevithas\}@iisc.ac.in.

Permission to make digital or hard copies of all or part of this work for personal or classroom use is granted without fee provided that copies are not made or distributed for profit or commercial advantage and that copies bear this notice and the full citation on the first page. Copyrights for components of this work owned by others than ACM must be honored. Abstracting with credit is permitted. To copy otherwise, or republish, to post on servers or to redistribute to lists, requires prior specific permission and/or a fee. Request permissions from permissions@acm.org.

(c) 2020 Association for Computing Machinery.

2639-0175/2020/07-ART25 \$15.00

https://doi.org/10.1145/3411756

Digital Government: Research and Practice, Vol. 1, No. 4, Article 25. Publication date: July 2020. 
timeline uniformly for all countries on an ad hoc basis, the website automatically detects phase of transmission in a data-centric manner for each country. Our analysis and website can be used to:

- Undertake comparative analysis among countries;

- Automatically detect and compare phases of spread across different countries;

- Prevent spread by taking examples from other countries.

In the following paragraphs, we list the existing visualization techniques and their shortcomings and then present our proposed approach with case studies of representative use cases.

\section{EXISTING VISUALIZATION TECHNIQUES}

A plethora of work has been done on visualizing COVID-19 data since the outbreak of the pandemic. Those visualizations can be primarily classified into two groups: visualization with zero or less interactivity represents the first group, and complex interactive visualization techniques and tools represents the second. Static visualization $[1,2]$ uses basic graphs such as bar and line graphs for representing attributes of COVID-19 dataset. The Washington Post [1] used a collection of bar graphs for comparing new cases and deaths between countries. Each chart represented a country and each bar of every chart displays the number of cases or deaths. The Financial Times developed a visualization [2] with a series of line charts for comparing daily deaths. Bar and line charts were combined together to form a composite static graph [3] for showing two different data attributes: bar chart was used to display number of new cases, with line charts showing the change of percentage of daily deaths. The chart was annotated with events such as schools closed, complete lockdown started, and so on. A few visualizations allowed user interaction $[4,5]$ like hovering the mouse over bars and points in line charts. Worldometers website was one such example that used two graphs for showing total number of cases and new cases every day. These types of representation are quite easy to implement and understand, but can miss relevant information. Two other visualizations [6] used more interactivity than the previous example with line charts to represent second derivative of daily number of cases and deaths displaying their progression rate. Majority of websites [1, 8-11] used interactive world map charts or world map globe as their primary layout for the visualization. Almost all these visualizations had hover and click interactivity displaying data attributes such as number of cases, number of deaths, active cases, recovered cases, number of fatal cases, and so on. Flourish studio [13] used four types of visualization techniques for managing distinct information with live updating graphics as follows:

(1) Interactive trajectory chart for number of cases and deaths;

(2) Sunburst was used for breaking the impact of virus by countries;

(3) Searchable line chart for number of confirmed cases and recovered cases;

(4) World map charts for number of cases and deaths.

Wikipedia and Tableau Public $[12,15]$ used interactive stacked bar charts for showing attributes such as active cases, total cases, deaths, recoveries, and number of people hospitalized. Interactive trajectory chart was used in International SOS website [14] to compare rates of new cases each day to determine how fast the outbreak is growing in countries. Users can toggle between logarithmic axes (default) and linear axes. This tool consists of two more charts: The first one compares between the number of cases against the number of tests performed, and the second one shows the cumulative tests carried out per 1,000 people in countries.

In summary, we have identified the following challenges while visualizing data related to the spread of the COVID-19 pandemic:

(1) Range of data: The number of cases and deaths range from a tenth of a million to less than a hundred in different states, regions, and countries. It is difficult to plot this wide range of values sharing a single scale. The trend may look flat even if it is not in countries or states where the number of new cases is in hundreds when plotted with a country or region where it is in thousands.

Digital Government: Research and Practice, Vol. 1, No. 4, Article 25. Publication date: July 2020. 
(2) Data transformation: Usually in science, researchers use log or semilog transformation for plotting data with a wide range. However, presently the COVID statistics have a huge influence on people's perception of threat, and a flat logarithmic curve may undermine the effect of the disease among common people if they do not follow the actual data transformation.

(3) Proportional statistics: A plethora of statistics is defining new variables such as number of people affected per capita or per million population or proportion of death with respect to total number of affected people. These statistics often exaggerate the problem for both small and large countries. For example, a huge number of deaths may seem a small proportion of per million people in countries such as India or China and only a few deaths may seem a big number for a small and less populous country.

(4) Information overload: When the graphs present data for multiple states or countries, all graphs turn indistinguishable at the early stage of the pandemic and countries where the spread has not yet reached peak clutter together even for a set of line graphs.

Difficulty in trend analysis: The WHO and Bing websites undertook a novel approach of relating the total number of cases with radius of a semi-transparent circle and placing it at geographic location. While this visualization can present a snapshot of present status, it does not provide an indication to trend of spread over time. The traditional trend analysis graphs are often not interactive and show a similar trend for many countries in a cluttered way.

\section{PROPOSED APPROACH}

We have proposed the following approach in visualizing the spread of the pandemic for comparison and prediction among different states and countries:

- Automatic phase detection: At present, the spread of COVID-19 is shown to have an exponential trend in most countries. An exponential curve is difficult for both comparison and prediction, as it has a vertical asymptotic trend to infinity. Our analysis automatically divides the duration of spread of the disease based on rate of increase in new cases using a knee detection algorithm $[16,17]$ and shows a set of three graphs that are easier to interpret and extrapolate than a single exponential graph. The shape of the graphs (such as linear, parabolic, or exponential) can be compared at different stages and countries with respect to the average number of new cases and deaths. We used two different $\mathrm{Y}$ axes to indicate number of cases and deaths.

- Combination of bar and line graphs: A line graph is good to indicate trend and takes least screen estate. However, if it is close to any axes, then the line often overlaps with the axis itself and may seem discontinuous. However, a column graph uses more screen estate and thus is more visible than a line graph while depicting a parameter with small values. When we plot both number of total cases and number of new cases per day using same $\mathrm{Y}$ axis, we used a line graph for total number of cases and a column graph for number of new cases per day. Same logic is also followed for showing number of deaths.

- Combination of line graph and pie chart: While three graphs are used for comparing different phases of spread for a single country or state, we have used a combination of line graph and pie chart (Figure 2) to compare the spread among multiple countries or states and their daily contributions to number of new cases and deaths. The line chart only compares the recent past among different countries or states. The pie chart shows the ratio of number of cases and deaths for a particular date of different countries or states with respect to the world or India, respectively.

- Comparative statistics on daily basis: For individual state or country, we divide the total duration in three separate graphs. Each of these three graphs (Figure 1) shows total number of cases and deaths as well as number of new cases and deaths per day. However, for comparison among multiple regions (Figure 2), we use only number of new cases and deaths per day for the following reasons: 




Date - US

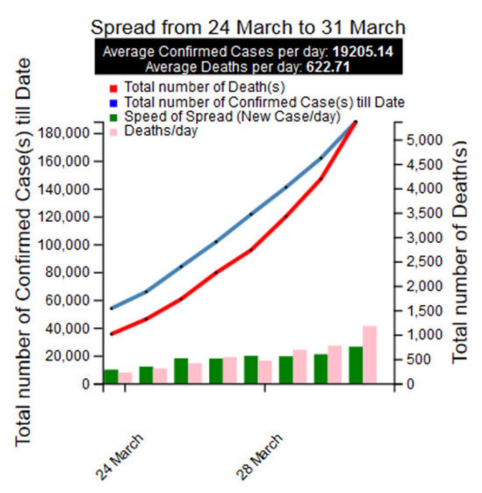

Date - US



Date - US

Fig. 1. Set of three graphs combining line and bar charts.

\section{Comparison of latest phase thorough automatic phase detection}
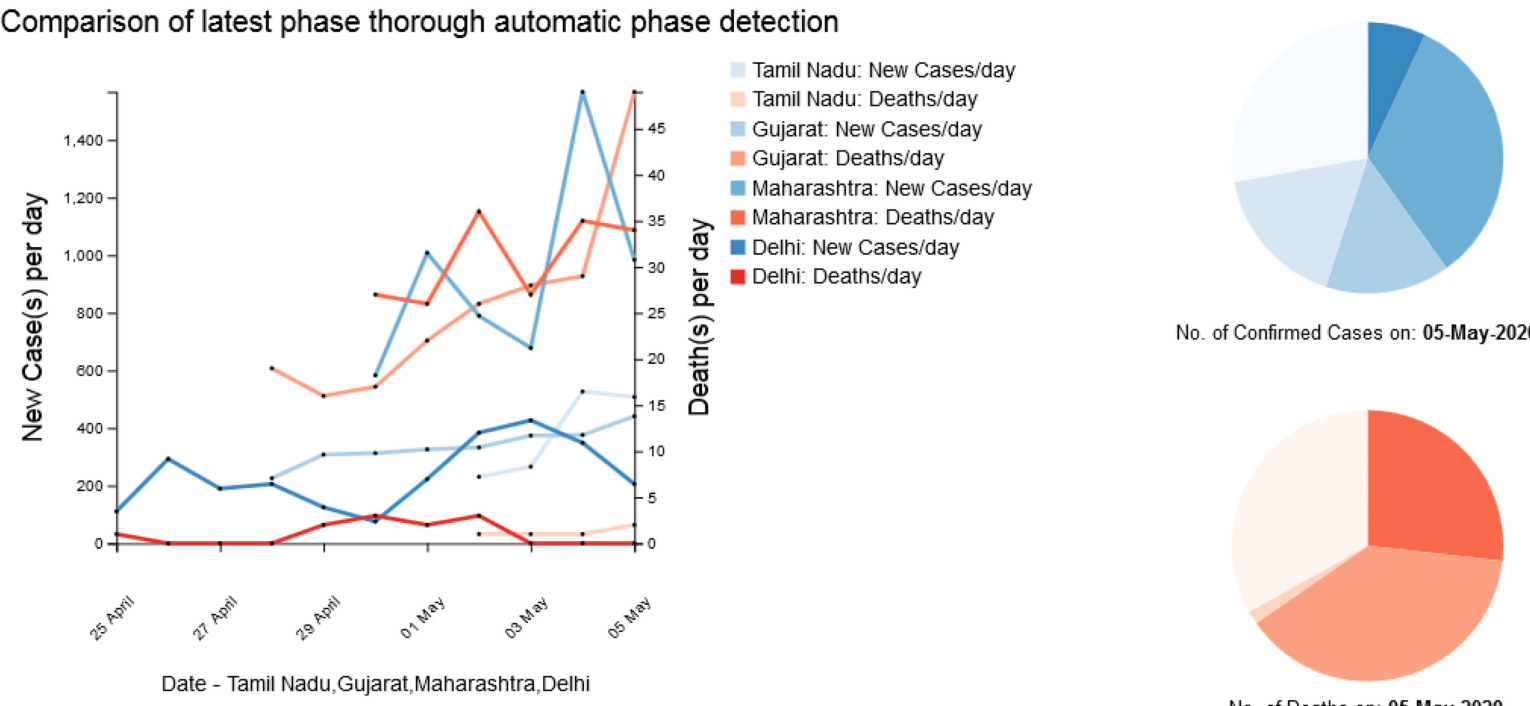

No. of Confirmed Cases on: 05-May-2020



No. of Deaths on: 05-May-2020

Fig. 2. Comparative analysis using line graph and pie chart.

- Number of new cases and deaths each day have a smaller range than total number of cases;

- Number of new cases and deaths per day depict the speed of spread and indicate the trend better than total number of cases.

A demonstration of the system can be found at https://youtu.be/3K9PnDFKKOE, while a demonstration of the system with automatic speech recognition and spoken output can be found at https://youtu.be/lyvwK9enFtw.

\section{IMPACT}

The website is developed for both common people who may not have detailed mathematical background to interpret a logarithmic or semi-log plot as well as for policy makers to quickly detect a rate of change of spread and take necessary action. For example, our automatic phase detection analysis (Figure 3) shows change in the rate of spread at different times in different Indian states on 27th May 2020. While the rate increased in 
Comparison of latest phase thorough automatic phase detection


No. of Deaths on: 27-May-2020

Fig. 3. Comparison of change in spread across Indian states.

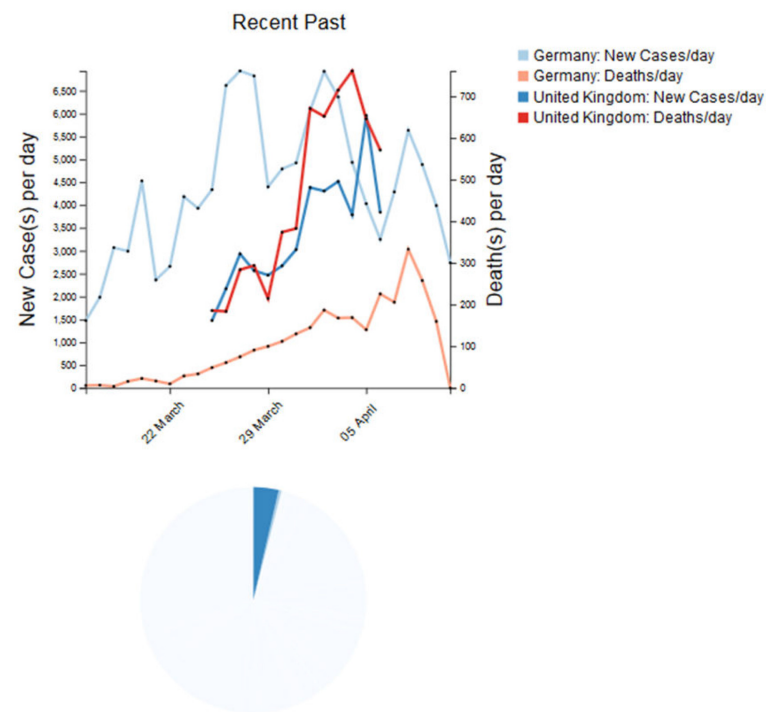

No. of Confirmed Cases on: 14-May-2620

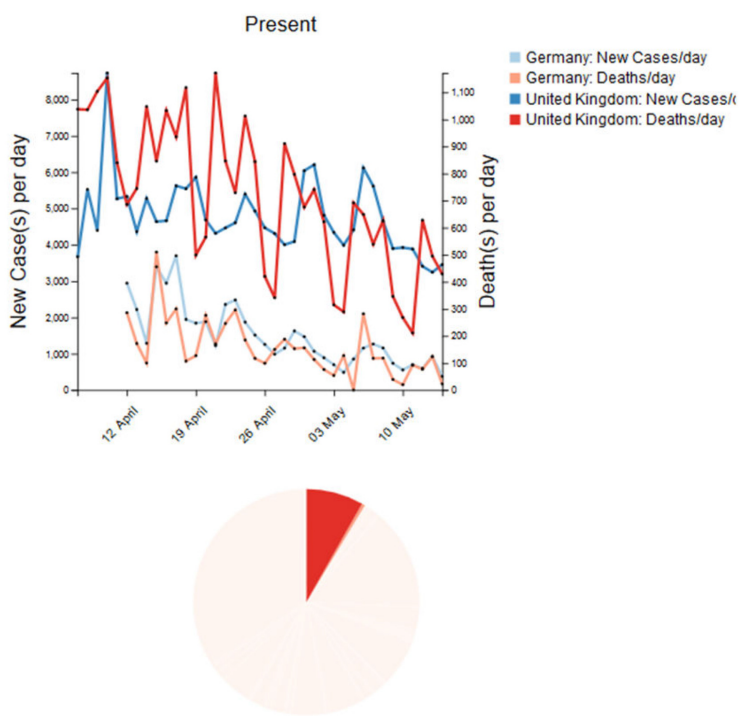

No. of Deaths on: 14-May-2020

Fig. 4. Comparative analyses at different phases between UK and Germany.

Maharashtra, Delhi, and Tamil Nadu in past 10 days, the rate remains same in Gujarat from start of May. Hence, policymakers can take appropriate actions for the states where the spread is increased recently.

Our website shows comparisons of recent past with present state of COVID-19 spread in multiple countries. For example, the following graphs generated on 14th May show that till mid-April, number of new cases was higher in Germany than UK although at present time the trend is reversed, as the pandemic is better contained in Germany than the UK (Figure 4). Policymakers can compare the policies adopted in the concerned countries and create new suggestions for countries such as India or Brazil where the pandemic is presently peaking. 


\section{Comparison of COVID -19 spread in India with European Countries on 21st May 2020}
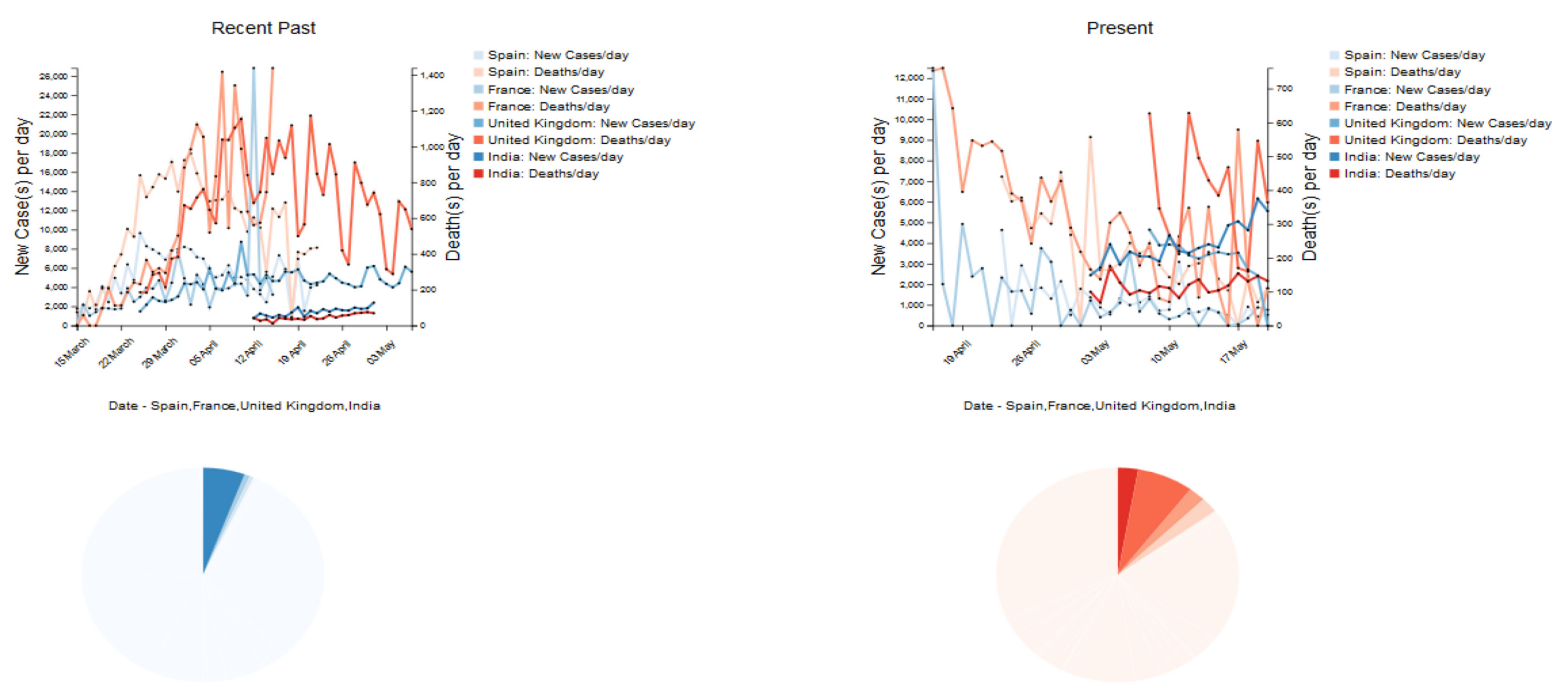

Fig. 5. Comparative analyses at different phases between India and European countries.

A similar analysis was undertaken on India and European countries on 21st May (Figure 5). It may be seen that India had much lower number of cases earlier but now the number of cases is higher than European countries and it is increasing. However, number of deaths is lower; it may be due to the lag in spread by which time medical facilities got more time to prepare. All graphs from Figures 1 to 5 can be generated on-the-fly from the website for any combination of countries. However, data collected from the website can also be used for post-processing as described in the next paragraph.

We undertook an exhaustive comparative analysis of all countries and states on 29th April and generated the following graph (Figure 6). The following graphs represent a comparison of average number of new cases per day across different countries and Indian states in recent past and present. The graphs should be magnified and read in clockwise direction from the top, which will indicate increasing trend. The green line and labels represent present while the red line and orange labels represent recent past. The Y-axis represents number of new cases per day.

Limitations: Our analysis is based on publicly available data and any anomaly or fault in data collection will also be reflected in our analysis. Our analysis visualizes publicly reported spread of the pandemic but we cannot infer anything about country-specific reporting and testing strategies. We did not divide the number of casualities/ deaths by total population or per capita income, as it may undermine the effect of the pandemic for bigger and high-income countries and add an extra variable in terms of comparison. We rather leave the choice to users to pick countries of interest and interpret data with respect to other factors such as testing rate, GDP, population, and so on.

\section{CONCLUSIONS}

This article summarizes existing visualization techniques used to visually represent spread of COVID-19 pandemic across different countries and presents a new interactive web-based system that automatically divides the duration of spread into three phases. A set of use cases demonstrates the utility of the new system in terms of comparing rate spread across different countries and different times. The system can also indicate rate and trend of spread over time and by comparing with past examples, the system can also be used to predict future rate of

Digital Government: Research and Practice, Vol. 1, No. 4, Article 25. Publication date: July 2020. 


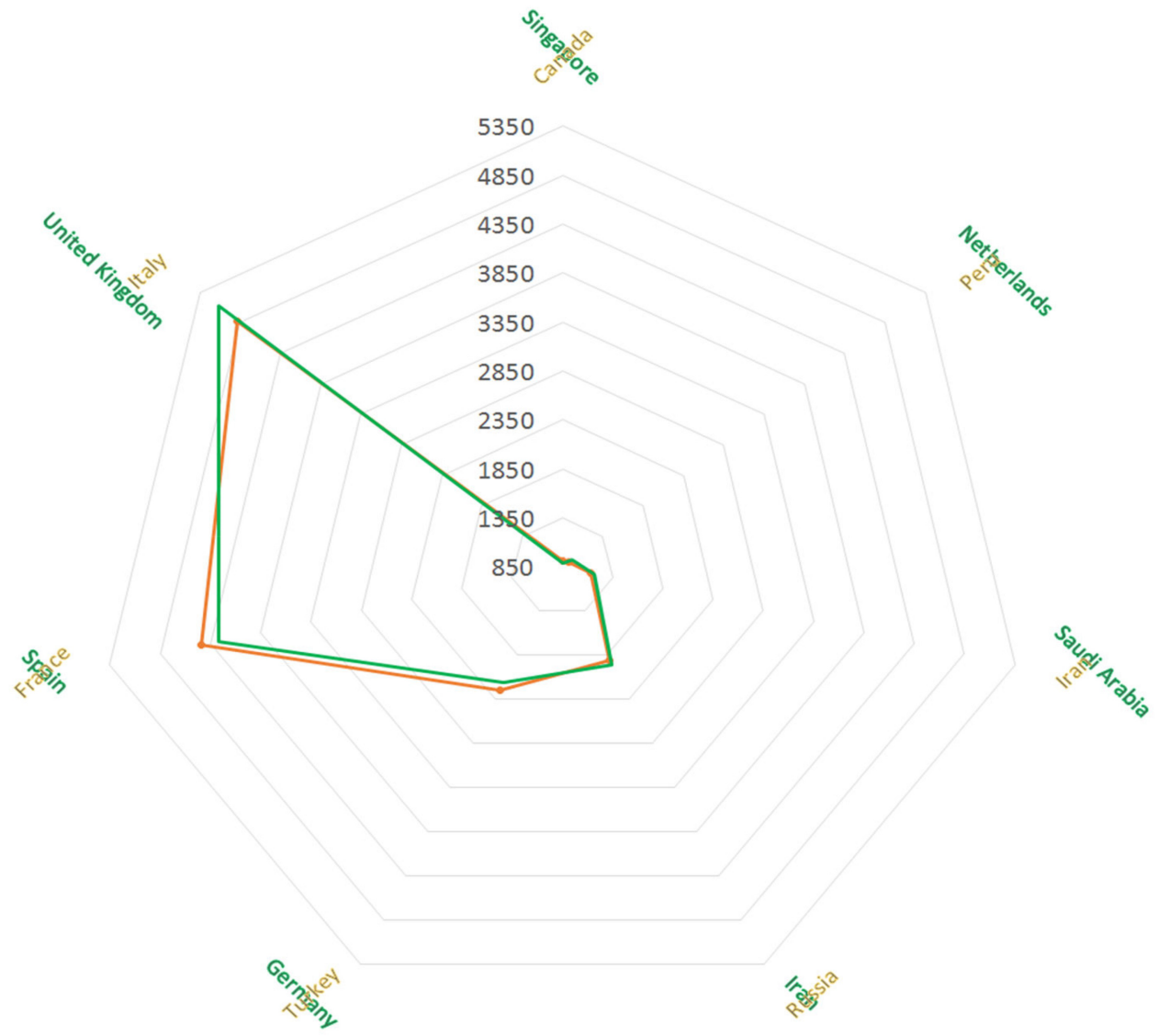

Fig. 6. Radar charts comparing rate of spread comparing countries where number of new cases per day is greater than 850 on 29th April.

spread. The system is also integrated to automatic speech recognition and text to speech features to disseminate information to people with different range of abilities.

\section{REFERENCES}

[1] Washington Post Staff. 2020. Mapping the Worldwide Spread of the Coronavirus. The Washington Post, WP Company (24 Apr. 2020). Retrieved from www.washingtonpost.com/graphics/2020/world/mapping-spread-new-coronavirus/.

[2] John Burn-Murdoch et al. 2020. Coronavirus Tracked: The Latest Figures as the Pandemic Spreads. Financial Times (25 Apr. 2020$)$. Retrieved from www.ft.com/coronavirus-latest.

[3] Kylie Stevens. 2020. Why Australia's "Suppression" Strategy Has Been More Effective in Flattening the Coronavirus Curve Than New Zealand's Complete Lockdown While Causing Less Economic Devastation. Daily Mail. (27 Apr. 2020). Retrieved from https://www.dailymail.co.uk/news/article-8259269/Australia-outperforming-New-Zealand-controlling-coronaviruscurve-without-stage-four-lockdown.html.

[4] Worldometer. 2020. Coronavirus Cases: Worldometer. Retrieved from www.worldometers.info/coronavirus/.

[5] Wade Fagen-Ulmschneider. 2020. 91-DIVOC. Flip the Script on COVID-19. Retrieved from 91-divoc.com/pages/covid-visualization/.

[6] D. Rémy. 2020. What's Wrong with COVID-19 Data Visualizations, and How to Fix It. Medium, Towards Data Science (5 Apr. 2020). Retrieved from towardsdatascience.com/whats-wrong-with-covid-19-data-visualizations-and-how-to-fix-it-3cdc9adc774d.

[7] MDN Web Docs. 2020. Webspeech API (28 Apr. 2020). Retrieved from https://developer.mozilla.org/en-US/docs/Web/API/Web_Speech_ API.

[8] World Health Organization. 2020. WHO Coronavirus Disease (COVID-19) Dashboard. Retrieved from covid19.who.int/.

Digital Government: Research and Practice, Vol. 1, No. 4, Article 25. Publication date: July 2020. 
[9] N. Mamoon and G. Rasskin. 2020. COVID-19 Visualizer. Retrieved from www.covidvisualizer.com/.

[10] Microsoft Bing. 2020. COVID-19 Tracker. Retrieved from bing.com/covid/local/india.

[11] COVID-19- Analysis, Visualization \& Comparisons. Kaggle, (7 Apr. 2020). Retrieved from https://www.cidrap.umn.edu/covid-19/mapsvisuals.

[12] Tableau Public. Retrieved from https://public.tableau.com/profile/oregon.health.authority.covid.19\#!/.

[13] Flourish. 2020. COVID-19: Free Live Mobile-Friendly Visualizations for Use on Any Website. Retrieved from flourish.studio/covid/.

[14] International SOS. 2020. Interactive Covid-19 Data by Location. Footer image. Retrieved from pandemic.internationalsos.com/2019ncov/covid-19-data-visualisation.

[15] Wikipedia, Wikimedia Foundation. 2020. COVID-19 Pandemic in India. (28 Apr. 2020). Retrieved from en.wikipedia.org/wiki/2020_ coronavirus_pandemic_in_India.

[16] V. Satopaa, J. Albrecht, D. Irwin, and B. Raghavan. 2011. Finding a "kneedle" in a haystack: Detecting knee points in system behavior. In Proceedings of the 31st International Conference on Distributed Computing Systems. 166-171.

[17] K. P. S. Saluja, J. S. Dv, S. Arjun, P. Biswas, and T. Paul. Analyzing eye gaze movement of users with different reading abilities due to learning disability. In Proceedings of the 3rd International Conference on Graphics and Signal Processing (ICGSP'19).

Received May 2020; revised June 2020; accepted June 2020

Digital Government: Research and Practice, Vol. 1, No. 4, Article 25. Publication date: July 2020. 